\title{
The lattice thermal conductivity of a semiconductor nanowire
}

\author{
Mei-Jiau Huang, ${ }^{\text {a) }}$ Wen-Yen Chong, and Tai-Ming Chang \\ Department of Mechanical Engineering, National Taiwan University, Taipei, Taiwan 106, \\ Republic of China
}

(Received 12 September 2005; accepted 14 April 2006; published online 15 June 2006)

\begin{abstract}
It has been found experimentally as well as theoretically that the lattice thermal conductivity can be largely reduced by the size confinement effect. The significant boundary scattering effect is one of the dominant factors. In most existing lattice thermal conductivity models, an empirical relation is used for this scattering rate. An unconfined or confined phonon distribution obtained based on the phonon Boltzmann equation and the relaxation time approximation is then employed to calculate the lattice thermal conductivity. In this work, we first attempt to derive an analytical form of the boundary scattering rate for phonon conduction in a semiconductor nanowire and then claim two reasonable ways to take it into account correctly. Consistent mathematical models in the sense that the effects of the size confinement on (i) the phonon dispersion relation, (ii) the phonon distribution, (iii) the phonon group and phase velocities, and (iv) the Debye temperature are finally proposed.

(C) 2006 American Institute of Physics. [DOI: 10.1063/1.2203721]
\end{abstract}

\section{INTRODUCTION}

The thermoelectric coolers have advantages over the traditional heat pumps because they are compact and highly reliable, have no moving parts, and use no refrigerants. The poor cooling efficiency of thermoelectric devices, which is limited by the material properties, however, limits their applications. The thermoelectric property of a material is characterized by its figure of merit, $Z=S^{2} \sigma / k$ where $S$ is the Seebeck coefficient, $\sigma$ is the electric conductivity, and $k$ is the thermal conductivity. ${ }^{1}$ The commonly known thermoelectric materials have $Z T$ values between about 0.6 and 1.0 at room temperature $T=300 \mathrm{~K}$. It is believed that practical applications could be many more if materials with ZT values $\approx 3$ could be developed. Recently, it has been found experimentally as well as theoretically ${ }^{2-8}$ that lowdimensional materials such as quantum wells, quantum wires, quantum dots, and superlattice structures have $Z T$ values much larger than their bulk counterparts. The increase in $Z T$ values is explained by the belief that reduced dimensionality changes the band structures (enhances the density of states near the Fermi energy), modifies the phonon dispersion relation, and increases the interface scattering of phonons. Consequently the electric resistance and the lattice thermal conductivity can be both reduced, particularly the latter. $^{7,8}$

Heat in semiconductors is mostly carried by acoustic phonons. Analytic thermal conductivity models are usually developed based on the Boltzmann transport equation and under the single mode relaxation time (SMRT) approximation. Commonly considered scattering mechanisms include the three-phonon Umklapp scattering, the mass-difference scattering, the phonon-electron scattering, the boundary scattering, and so on. ${ }^{9}$ The Matthiessen's rule is then employed to sum up these scattering rates. Above all, two boundary effects have been taken into consideration. One is the modi-

${ }^{a)}$ Electronic mail: mjhuang@ntu.edu.tw fication of the phonon dispersion due to the spatial confinement and the other is the change in the nonequilibrium phonon distribution due to the partially diffuse boundary scattering. The former results in smaller group velocity as well as phase velocity. The latter corrects the formula for calculating the lattice thermal conductivity.

For instance, Khitun et al. ${ }^{10}$ computed the acoustic phonon dispersion relations of free-surface and clamped-surface nanowires based on the elasticity equation. ${ }^{11}$ The boundary scattering rate was estimated as the Casimir limit ${ }^{12}$ and the lattice thermal conductivity was computed in use of the unconfined phonon distribution. A similar work was performed for freestanding semiconductor thin film by Balandin and Wang (1998). ${ }^{6}$ Hyldgaard and Mahan (1996) (Ref. 13) calculated the in-plane thermal conductivity of GaAs/AlAs superlattices. They assumed a cubic isotropic bulk dispersion relation and calibrated the model parameters by the measured longitudinal and transverse Debye temperatures and by the maximum wave number (determined by the lattice constant) of GaAs. Lattice thermal conductivity was computed via the unconfined as well as the confined phonon distribution for thin-film structures. Walkauskas et al. (1999) (Ref. 14) applied a similar work to GaAs nanowires. Chung et al. (2004) (Ref. 15) explored the influence of the phonon dispersion relation. Five different dispersion relations were adopted. They found that phonon dispersion is required for the sake of eliminating the nonphysical discontinuity in the transverse phonon relaxation time. Dispersion relations for phonons were also computed using the method of valenceforce potentials. Kiselev et al. (2000) (Ref. 16) employed a so-obtained dispersion relation in calculating the lattice thermal conductivity via a bulk formula.

Noticing that the previous theories handled either the confined dispersion relation or the confined phonon distribution but not both simultaneously, Zou and Balandin (2001) (Ref. 17) took care of both effects and studied the differences in various combinations. The boundary scattering effect, however, was doubly counted in their work. It was counted 
when they estimated the total scattering rate and again when they solved the confined phonon distribution. Through an analogy of the equation for the phonon intensity (flux of energy per unit time, per unit area, per unit solid angle in the direction of phonon propagation and per unit frequency interval) with the equation of radiative transfer, ${ }^{18}$ Chen (1997) (Ref. 19) solved the phonon distribution in semiconductor superlattices under the SMRT approximation and calculated the in-plane thermal conductivity using a sine dispersion relation. In $1998,{ }^{20}$ he extended the work to analyze the crossplane thermal conductivity but adopting a linear dispersion relation.

It is worth mentioning that, strictly speaking, phonons of a quantized mode can be no longer viewed as approaching the nanostructure surface but phonons of a superposition of modes can. Among those models mentioned above, that involve the confined dispersion relation (or the quantized phonon modes), an average phonon dispersion relation over those quantized phonon modes having the same frequency is usually made and used as if it were three dimensional and isotropic. In other words, the spatial confinement effect on the phonon spectrum is modeled simply through a replacement of the bulk dispersion relation by the average one.

Although most of the lattice thermal conductivity theories mentioned above can predict qualitatively and quantitatively reasonably well the lattice thermal conductivity, none of them has taken a full consideration of the spatial confinement effects. A full consideration, to the authors' knowledge, includes a consideration of not only the two boundary effects mentioned above but also the difference between the group velocity and the phase velocity as well as the possible changes in the Debye temperatures. This constitutes the motivation of the present investigation. Moreover, an analytical (instead of empirical) boundary scattering rate is so far not attempted and will be developed herein. To avoid doubly counting it, two possible formulations will be proposed and compared.

The rest of this paper is arranged as follows. In the next section, we present all the associated theories. Results and discussions are given in Sec. III. Conclusions are in Sec. IV.

\section{PHONON BOLTZMANN EQUATION}

Consider a semiconductor nanowire of diameter $D$ which is on the order of phonon mean free path. We start the analysis with the steady time-relaxation-approximated phonon Boltzmann equation, namely,

$$
\mathbf{V} \cdot \nabla n=\frac{n_{0}-n}{\tau}
$$

where $\mathbf{V}$ is the phonon group velocity (in general a function of the wave vector $\mathbf{q}$ ), $n$ is the phonon distribution, and $n_{0}$ is the equilibrium (Planck) distribution at the local temperature $T$, that is

$$
n_{0}=\frac{1}{\exp \left(\hbar \omega / k_{B} T\right)-1} .
$$

The phonon distribution is usually written as $n=n_{0}-\tilde{n}$ and consequently $\tilde{n}$ satisfies

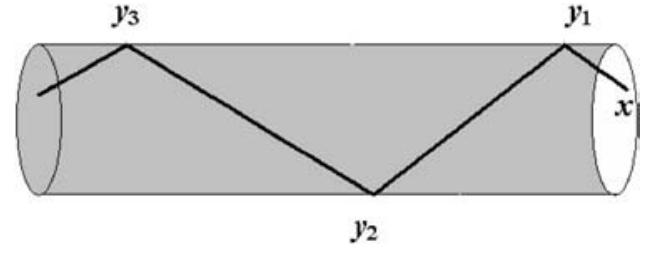

FIG. 1. An illustration of the pathline of a traveling phonon in a nanowire.

$$
\mathbf{V} \cdot \nabla \tilde{n}+\frac{\tilde{n}}{\tau}=\mathbf{V} \cdot \nabla n_{0}
$$

To solve Eq. (3), we need to estimate the total scattering rate, $1 / \tau$. The phonon scattering mechanisms considered by Zou et $a l .{ }^{17}$ are reconsidered herein. In the present study, models of the scattering rates due to the Umklapp process $\left(1 / \tau_{U}\right)$, the mass-difference scattering $\left(1 / \tau_{M}\right)$, and the phononelectron scattering $\left(1 / \tau_{\text {ph-e }}\right)$ are taken directly from the work of Zou et al. ${ }^{17}$ The boundary scattering rate $\left(1 / \tau_{B}\right)$, however, will be derived analytically in a way similar to that used by Casimir $^{12}$ as described in the following subsection. Finally, the total scattering rate is obtained by using the Mathiessen's rule,

$$
\frac{1}{\tau}=\frac{1}{\tau_{U}}+\frac{1}{\tau_{M}}+\frac{1}{\tau_{B}}+\frac{1}{\tau_{\text {Ph-e }}} .
$$

Silicon nanowires are of interest and the model parameters and material properties are all extracted from the work of Zou et al.. ${ }^{17}$

\section{A. Boundary scattering rate}

In order to compute the boundary scattering rate, all the other scattering mechanisms will be ignored temporarily. The phonon Boltzmann equation is thus reduced to

$$
\mathbf{V} \cdot \nabla \tilde{n}=\mathbf{V} \cdot \nabla n_{0} .
$$

Let $p$ be the fraction of specularly reflected phonons at the boundary. It is easy to show that the solution of Eq. (5) can be written as ${ }^{12}$

$$
\tilde{n}(\mathbf{q}, \mathbf{x})=\frac{\partial n_{0}}{\partial T} \nabla T \cdot(1-p) \sum_{j=1}^{\infty} p^{j-1}\left(\mathbf{x}-\mathbf{y}_{j}\right),
$$

where $\mathbf{y}_{j}$ 's are the successively hitting points at the boundary along the "specular" phonon pathline as shown in Fig. 1. An assumption has been made and employed in Eq. (6) nonetheless that the temperature gradient is so small that the variations of the local equilibrium distribution along the phonon pathline are negligible. Noticing

$$
\nabla T \cdot\left(\mathbf{x}-\mathbf{y}_{1}\right)=\xi \cot \theta|\nabla T|
$$

and

$$
\nabla T \cdot\left(\mathbf{y}_{j}-\mathbf{y}_{j+1}\right)=D \cot \theta|\nabla T|,
$$

one can rewrite Eq. (6) as 


$$
\tilde{n}(\mathbf{q}, \mathbf{x})=\frac{\partial n_{0}}{\partial T}|\nabla T|(1-p)\left(\xi \cot \theta \sum_{i=0}^{\infty} p^{i}+D \cot \theta \sum_{i=1}^{\infty} i p^{i}\right)
$$

or

$$
\widetilde{n}(\mathbf{q}, \mathbf{x})=\frac{\partial n_{0}}{\partial T}|\nabla T|\left(\xi \cot \theta+D \cot \theta \frac{p}{(1-p)}\right),
$$

where $\xi$ is the projection length of the vector $\left(\mathbf{x}-\mathbf{y}_{1}\right)$ onto the cross section of the nanowire and $\theta$ is the angle between the phonon group velocity and the axis of the nanowire.

Next, we recall that

$$
k=\frac{1}{3} C \bar{V} \Lambda
$$

where $C$ is the specific heat, $\bar{V}$ is some overall averaged phonon group velocity, and $\Lambda$ is the mean free path. Also recalled is the heat flux averaged over the cross section $A$ $=\pi D^{2} / 4$ of the nanowire which is computed as

$$
q^{\prime \prime}=-\frac{1}{(2 \pi)^{3} A} \iint \tilde{n} \hbar \omega \mathbf{V} \cdot d \mathbf{A} d \mathbf{q} .
$$

According to Fourier's law, one may write the mean free path as follows:

$$
\Lambda=\frac{3}{|\nabla T|}\left(\int \frac{\partial n_{0}}{\partial T} \hbar \omega V d \mathbf{q}\right)^{-1}\left(\frac{1}{A} \iint \tilde{n} \hbar \omega \mathbf{V} \cdot d \mathbf{A} d \mathbf{q}\right),
$$

where

$$
C \bar{V}=\frac{\partial}{\partial T} \frac{1}{(2 \pi)^{3}} \int n \hbar \omega V d \mathbf{q} \approx \frac{1}{(2 \pi)^{3}} \int \frac{\partial n_{0}}{\partial T} \hbar \omega V d \mathbf{q}
$$

has been adopted. Substituting Eq. (9) into Eq. (12), one obtains

$$
\Lambda=\frac{3}{4 \pi A} \int_{A} \int_{0}^{2 \pi} \int_{0}^{\pi}\left(\xi+\frac{D p}{(1-p)}\right) \cos ^{2} \theta d \theta d \phi d A .
$$

Using

$$
\int_{A} d A=\frac{1}{2} \int_{0}^{D} \sqrt{D^{2}-\xi^{2}} d \xi
$$

and performing all the integrations, after some algebra, one finally finds

$$
\Lambda=D\left(1+\frac{p}{(1-p)} \frac{3 \pi}{4}\right) .
$$

Equation (15) suggests that the boundary scattering rate can be modeled as

$$
\frac{1}{\tau_{B}}=\frac{V}{\Lambda}=\frac{V}{D}\left(1+\frac{p}{(1-p)} \frac{3 \pi}{4}\right)^{-1} .
$$

It is easy to check that this rate turns to be zero when $p=1$ and $V / D$ (the Casimir limit) when $p=0$. Moreover, compared to the commonly used empirical relation

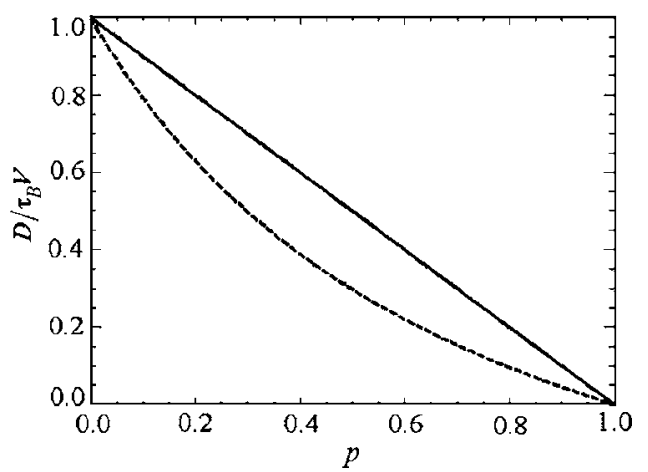

FIG. 2. The empirical (solid line) and the analytical (dashed line) boundary scattering rates.

$$
\frac{1}{\tau_{B}}=\frac{V}{D}(1-p)
$$

the newly proposed one is weaker as shown in Fig. 2.

\section{B. Lattice thermal conductivity formula}

To take the boundary scattering effect into consideration, two possible reasonable formulations are discussed herein. The first one is to treat it as a part of the total scattering rate; that is, one solves Eq. (3) by adopting Eq. (4) and pretending no existence of the boundaries. Therefore, the (bulk) formula is also usable for the semiconductor nanowires, namely,

$$
k_{l}=\frac{1}{6 \pi^{2}} \frac{k_{B}^{4} T^{3}}{\hbar^{3}} \sum_{i} \int_{0}^{\theta_{D} / T} \frac{V \tau}{V_{p}^{2}} \frac{x^{4} e^{x}}{\left(e^{x}-1\right)^{2}} d x,
$$

where the summation is done over the polarizations, $V_{p}$ $=\omega / q$ is the phonon phase velocity (in general different from the group velocity $V=d \omega / d q), \theta_{D}$ is the Debye temperature, and $\hbar$ and $k_{B}$ are the Planck constant and the Boltzmann constant, respectively. Although the same formula is involved for bulk materials and nanowires, the so-computed thermal conductivities, however, are different because of the difference in the dispersion relations, the difference in the Debye temperatures, and an additional boundary scattering rate necessary for nanowires.

The second possible way to handle the boundary scattering effect is to solve exactly Eq. (3) with partially diffuse boundaries and under the existence of all other scattering mechanisms. For semiconductor nanowires of diameter $D$, the associated phonon distribution is well known and the formula for computing the lattice thermal conductivity is modified as follows: ${ }^{13,17}$

$$
\begin{aligned}
& k_{\text {wire }}=k_{l}-\Delta k, \\
& \Delta k=\frac{2}{\pi^{3}} \frac{k_{B}^{4} T^{3}}{\hbar^{3}} \sum_{i} \int_{0}^{\theta_{D} / T} G(\eta) \frac{V \tau}{V_{p}^{2}} \frac{x^{4} e^{x}}{\left(e^{x}-1\right)^{2}} d x,
\end{aligned}
$$

where $\eta=D / \tau V$ and the function $G(\eta)$ is defined as

$$
G(\eta)=(1-p)^{2} \sum_{j=1}^{\infty} j p^{j-1} \int_{0}^{1} \sqrt{1-\xi^{2}} S_{4}(j \eta \xi) d \xi
$$

with 


$$
S_{4}(u)=\int_{0}^{\pi / 2} \exp \left(-\frac{u}{\sin \theta}\right) \cos ^{2} \theta \sin \theta d \theta .
$$

Again the variation of the local equilibrium distribution along the phonon pathline has been ignored. It must be very careful now, however, when the overall relaxation time $\tau$ is evaluated. Because the boundary scattering effect has been taken into consideration in deriving the phonon distribution, it should be excluded from the total scattering rate, i.e., now

$$
\frac{1}{\tau}=\frac{1}{\tau_{U}}+\frac{1}{\tau_{M}}+\frac{1}{\tau_{\text {ph-e }}} .
$$

To be consistent, it should be kept in mind that the phonon velocities remain functions of frequency $\omega$ at all times in the two formulations. From now on, the first method will be called the A model and the second one will be called the $\mathrm{B}$ model for convenience.

\section{Dispersion relations}

The phonon dispersion relations for isotropic continuum materials are considered. For bulk materials, the group velocities are constant and the dispersion relations are linear: $\omega=s_{l} q$ for the longitudinal polarization and $\omega=s_{t} q$ for the transverse polarization. In the case of free-surface semiconductor nanowires, two axisymmetric polarizations have been reported. ${ }^{11}$ The confined polarization is a mixed mode of the longitudinal and transverse polarizations. Its dispersion relation is determined by ${ }^{11}$

$$
\begin{aligned}
& 4 q^{2} q_{l}^{2} \frac{\left(q_{t} D / 2\right) J_{0}\left(q_{t} D / 2\right)}{J_{1}\left(q_{t} D / 2\right)}-2 q_{l}^{2}\left(q^{2}+q_{t}^{2}\right) \\
& +\left(q^{2}-q_{t}^{2}\right)^{2} \frac{\left(q_{l} D / 2\right) J_{0}\left(q_{l} D / 2\right)}{J_{1}\left(q_{l} D / 2\right)}=0, \\
& \omega^{2}=s_{l}^{2}\left(q^{2}+q_{l}^{2}\right)=s_{t}^{2}\left(q^{2}+q_{t}^{2}\right) .
\end{aligned}
$$

The other polarization is a pure transverse polarization having its dispersion relation determined by

$$
\begin{aligned}
& J_{0}\left(\frac{q_{\theta} D}{2}\right)-\frac{4}{q_{\theta} D} J_{1}\left(\frac{q_{\theta} D}{2}\right)=0, \\
& \omega^{2}=s_{t}^{2}\left(q^{2}+q_{\theta}^{2}\right) .
\end{aligned}
$$

Both polarizations have infinitely many and discrete solutions for a given wave number $q$ as shown in Figs. 3 and 4 for a silicon nanowire. For simplification, the concept of the virtual energy levels is usually employed, ${ }^{6}$ which assumes that the phonon modes corresponding to a given frequency $\omega$, as shown in Fig. 3, possess energies of $s \hbar \omega$, for $s$ $=12, \ldots$. A so-called "population-weighted" average group velocity corresponding to the frequency $\omega$ is thus defined as

$$
V(\omega) \equiv \frac{\sum_{s} V_{s} e^{-s \hbar \omega / k_{B} T}}{\sum_{s} e^{-s \hbar \omega / k_{B} T}},
$$

where $V_{s}$ is the group velocity of the $s$ th mode. Nonetheless, since all these modes have the same frequency (equivalently

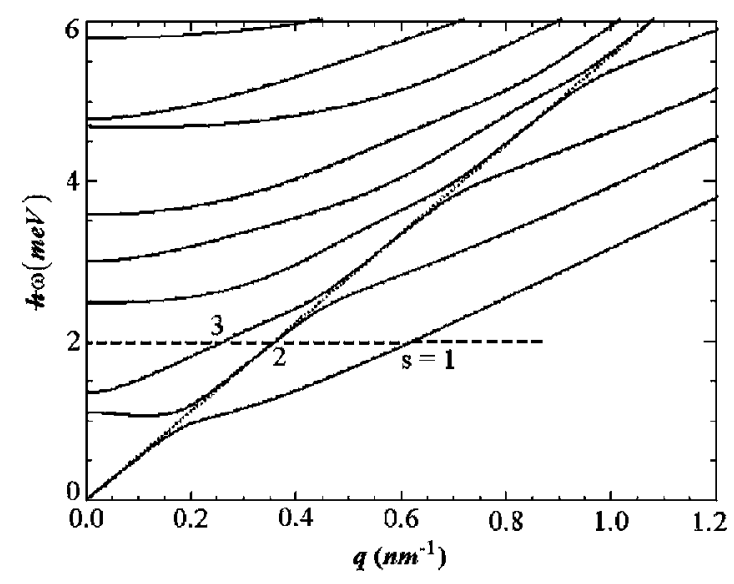

FIG. 3. The eigenmodes of the confined polarization of a silicon nanowire $(D=20 \mathrm{~nm})$. The dotted line is the linear (bulk) dispersion relation of the longitudinal polarization.

the same energy), an algebraic average of the group velocity is seemingly more reasonable. Both averages are calculated and shown in Fig. 5. It is seen that both averages of the confined polarization at large frequencies are smaller than the bulk transverse velocity $\left(s_{t}=5.34 \mathrm{~km} / \mathrm{s}\right)$. This is because the lowest mode $(s=1)$ of the confined polarization has a smaller group velocity $(4.81 \mathrm{~km} / \mathrm{s})$ and is weighted strongly at large frequencies. The last small bump in the populationweighted velocity at large frequencies arises from the contributions of some high modes having their velocities close to the bulk longitudinal velocity $\left(s_{l}=8.47 \mathrm{~km} / \mathrm{s}\right)$. The weights of these modes and consequently their contributions gradually decrease nonetheless as the frequency further increases. No such bump is observed on the other hand in the algebraic average. It must be because the last modes have negligible velocities and possess the same weight like others. They smooth out the larger velocities, resulting in a more rapid convergence. For a similar reason, the algebraic average group velocity of the transverse polarization is smaller than the population-weighted one, which converges to the bulk transverse velocity. It can be expected now that the lattice thermal conductivity calculated based on the algebraic average group velocity is also smaller. Because it is more physically reasonable, the algebraic average will be adopted in the present study.

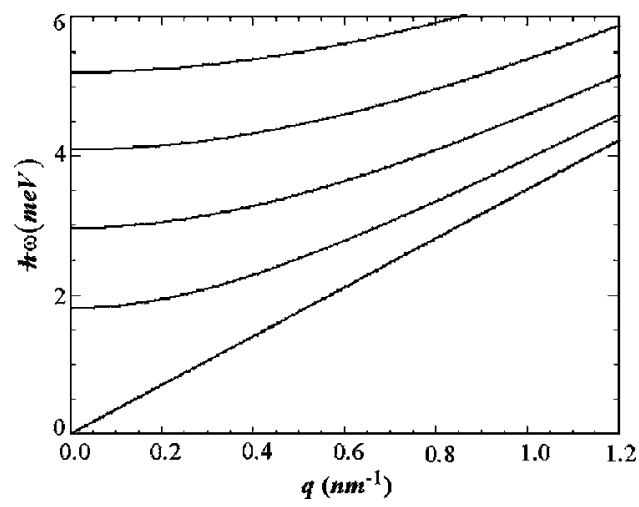

FIG. 4. The eigenmodes of the transverse polarization of a silicon nanowire $(D=20 \mathrm{~nm})$. The dotted line is the linear (bulk) dispersion relation of the transverse polarization, which is hardly distinguished from the lowest mode. 

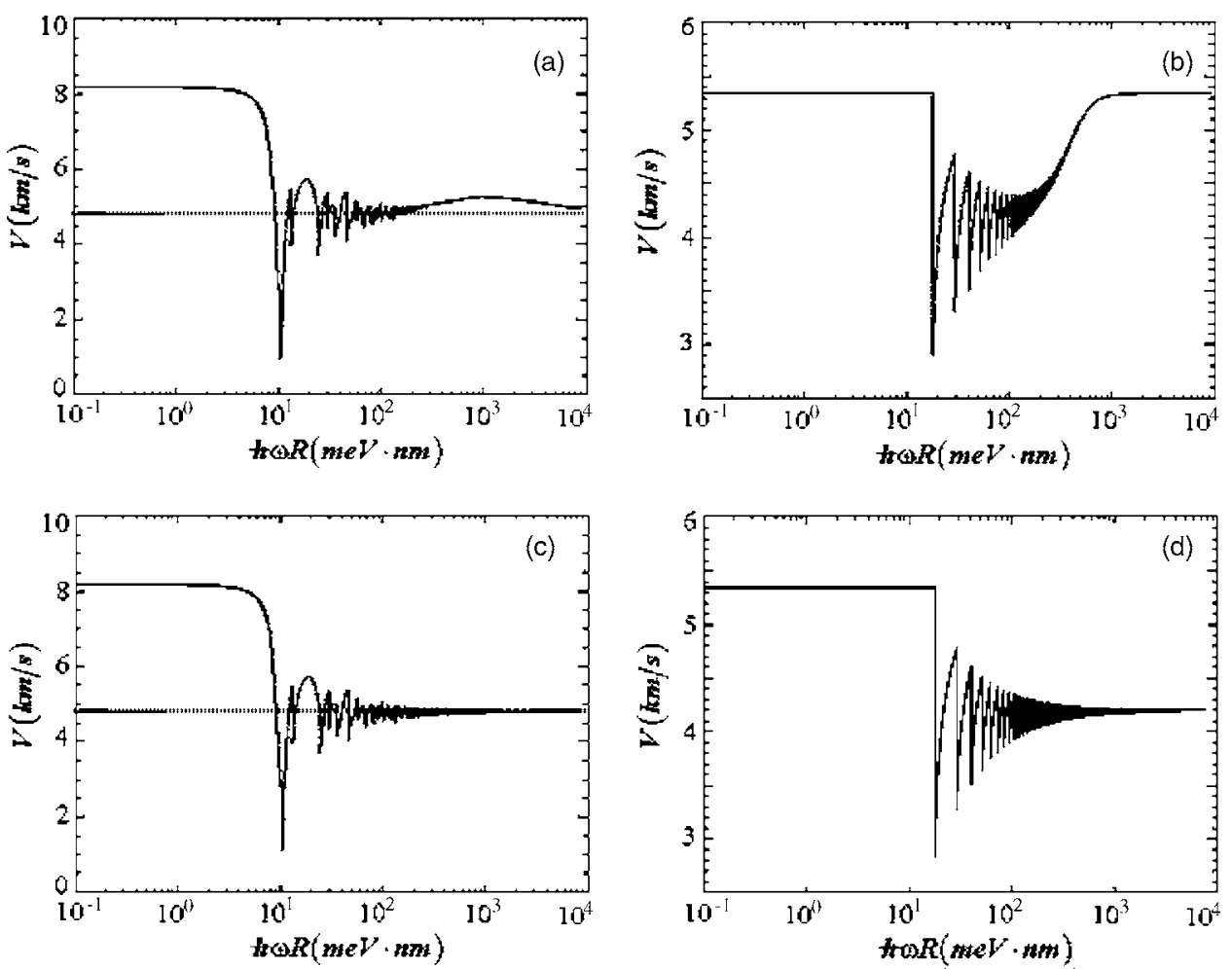

FIG. 5. The population-weighted averages $[(a)$ and (b)] and the algebraic averages [(c) and (d)] of the confined [(a) and (c)] and the transverse [(b) and (d)] polarizations.
A unique dispersion relation for each polarization can now be obtained by integrating

$$
V(\omega) d q=d \omega .
$$

Finally, it should be mentioned that as one longitudinal and two transverse polarizations are counted for bulk materials, two confined polarizations and one transverse polarization are included when nanowires are of interest.

\section{Debye temperature}

In most of the previous investigations of the lattice thermal conductivity, same Debye temperatures are used for both bulk and low-dimensional materials. However, because the dispersion relations have been modified due to the spatial confinement effect, the Debye temperature may be changed as well. If the crystal of interest is assumed to be isotropic and $a$ is the associated lattice constant, according to the Debye model, ${ }^{9,13}$ the cutoff wave number $q_{D}$ (for silicon) is related to the lattice constant by

$$
\frac{4 \pi}{3} q_{D}^{3}=4(2 \pi)^{3} / a^{3} .
$$

In this study, we attempt to adopt this cutoff wave number and determine the associated Debye frequency using the dispersion relations obtained in the previous subsection, i.e., $\omega_{D}=\omega\left(q_{D}\right)$. The Debye temperature $\theta_{D}=\hbar \omega_{D} / k_{B}$ is obtained then.

\section{RESULTS AND DISCUSSIONS}

Take silicon nanowires for illustration. The lattice constant of silicon is $5.43 \AA$. From Eq. (30), the cutoff wave number is found to be $11.4 \mathrm{~nm}^{-1}$. The corresponding Debye frequencies are $\hbar \omega_{D}=36.2 \mathrm{meV}$ for the confined polarization and $31.7 \mathrm{meV}$ for the transverse polarization of the nanowires with diameter of $20 \mathrm{~nm}$. As far as bulk materials are concerned, the Debye frequencies are found to be 63.6 and $40 \mathrm{meV}$ for the longitudinal polarization and transverse polarization, respectively. The corresponding Debye temperatures of nanowires are thus 420 and $368 \mathrm{~K}$, smaller than those of bulk materials, namely, 738 and $465 \mathrm{~K}$. Because the upper limits of the integrations in Eqs. (18) and (20) are proportional to the Debye temperatures and because the scattering rate due to the Umklapp process is inversely proportional to the Debye temperature, ${ }^{17}$ a reduction in the lattice thermal conductivity of a silicon nanowire is therefore expected, not only due to smaller phonon group velocities and the additional boundary scattering rate but also due to decreased Debye temperatures.

Shown in Fig. 6 are the lattice thermal conductivities

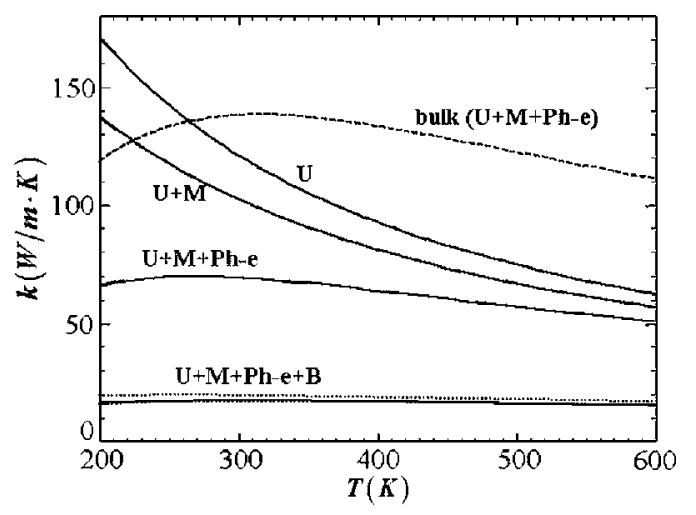

FIG. 6. The lattice thermal conductivity of a silicon nanowire with diameter of $20 \mathrm{~nm}$ with perfectly diffuse boundaries $(p=0)$ against the temperature when different scattering mechanisms are considered. The dashed curve is the bulk lattice thermal conductivity. The solid curves are predictions from the A model and the dotted curves are from the B model. 


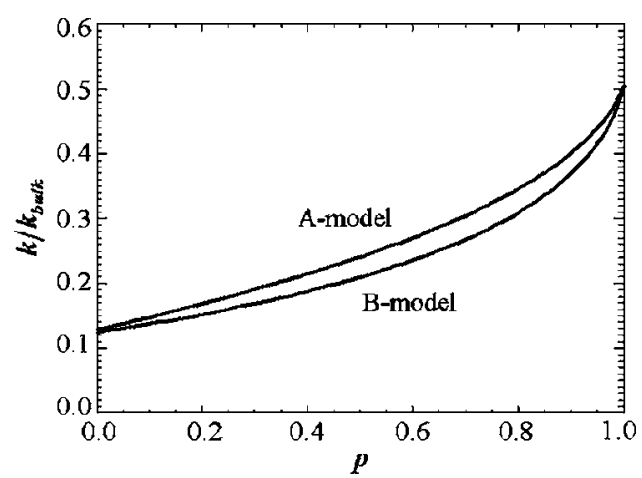

FIG. 7. The lattice thermal conductivity of a silicon nanowire with diameter of $20 \mathrm{~nm}$ against the specular fraction $p$ at $T=300 \mathrm{~K} \quad\left(k_{\text {bulk }}\right.$ $=138.3 \mathrm{~W} / \mathrm{m} \mathrm{K})$.

predicted by both the A and B models, compared to the bulk ones against the temperature. The diameter of the nanowire is $20 \mathrm{~nm}$ and the boundary is assumed perfectly diffuse ( $p$ $=0$ ). From the results of the A model, it is seen that the lattice thermal conductivity of the nanowire is about half of the bulk value when the boundary scattering rate has not be considered. This reduction is obviously caused by the changes in the dispersion relations. As the boundary scattering effect is taken into consideration, the lattice thermal conductivity is further reduced and remains nearly temperature independent. On the other hand, when the boundary scattering effect is taken into account explicitly in the formula (the B model), the other three scattering mechanisms show little net effect. They must cause a close reduction in $k_{l}$ as well as $\Delta k$. Both models nonetheless predict nearly the same results when all scattering effects are considered, and moreover these results are close to those obtained by Zou $e t$ al. ${ }^{17} \mathrm{Sev}-$ eral differences, however, are existent between the present models and theirs. In the work of Zou et al. ${ }^{17}$ the following have been done: only the contribution of the confined polarization to the lattice thermal conductivity was computed and directly tripled to replace the summation over polarizations; the same Debye temperature $625 \mathrm{~K}$ was employed for both the bulk and nanowire cases; the empirical relation for the boundary scattering rate, namely, Eq. (17), was used; the modified formula, Eq. (19), and the boundary scattering rate $1 / \tau_{B}$ are used simultaneously; and finally an overall mean phonon group velocity is used to replace both the frequencydependent group and phase velocities in the formulas.

The influence of the specular fraction $p$ is investigated in Fig. 7. With no surprise, the lattice thermal conductivity increases with increasing specular fraction. As the boundary is perfectly specular $(p=1)$, both $1 / \tau_{B}$ and $\Delta k$ vanish. The computed lattice thermal conductivity, however, is still smaller than the bulk value because the dispersion relations are different. The rapid decrease in the lattice thermal conductivity as $p$ is slightly smaller than 1 implies the powerfulness of the boundary scattering effect.

As the diameter of the nanowire increases, so is the lattice thermal conductivity, mainly because the boundary scattering rate decreases. This is illustrated in Fig. 8. Theoretically, it should get saturated gradually and approach to the bulk value. It does not, however, in the present models and

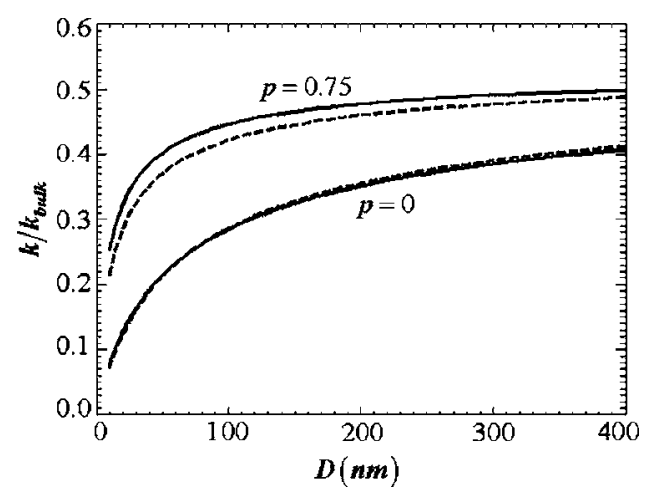

FIG. 8. The lattice thermal conductivity of a silicon nanowire against the diameter $D$ at $T=300 \mathrm{~K}\left(k_{\text {bulk }}=138.3 \mathrm{~W} / \mathrm{m} \mathrm{K}\right.$; solid line: A model; dashed line: B model).

neither in all the previous theories which employ the averaged dispersion relations over the infinitely many discrete eigenmodes (Figs. 3 and 4). The problem is that neither the confined nor the transverse polarization can be recovered to become a longitudinal polarization by simply increasing the diameter. These models therefore are not valid for very large diameters. They are not valid either when the diameter is smaller than or comparable to the phonon coherence length due to the appearance of wave characteristics. There, however, exists a middle regime in which the phonons travel at confined velocities and are significantly affected by the boundary scattering. In Fig. 9 we plot the deduction percentages of lattice thermal conductivity by the boundary scattering, namely, $\Delta k / k_{l}$ (in the A model, $k_{l}$ is defined as the lattice thermal conductivity computed when the boundary scattering is absent and $\Delta k$ is the difference when the boundary scattering is absent or present). This ratio evaluates the importance of the boundary scattering and may be helpful in estimating the validity of the models. As seen, approximately beyond $D=200 \mathrm{~nm}$, the deduction is below $10 \%$ when $p$ $=0.75$. A small deduction implies nonsignificant spatial confinement effect. In that case, the models discussed above are probably no longer valid and a traditional Knudsen theory with unconfined phonon dispersion relation should be expected.

From Figs. 6-8, it is observed that predictions from the $\mathrm{B}$ model are smaller than those from the A model in general.

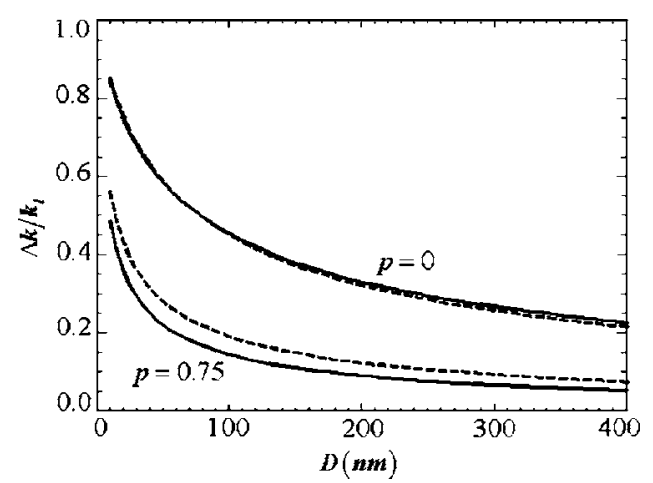

FIG. 9. The deduction percentages of lattice thermal conductivity by the boundary scattering at $T=300 \mathrm{~K}$ (solid line: A model; dashed line: B model). 


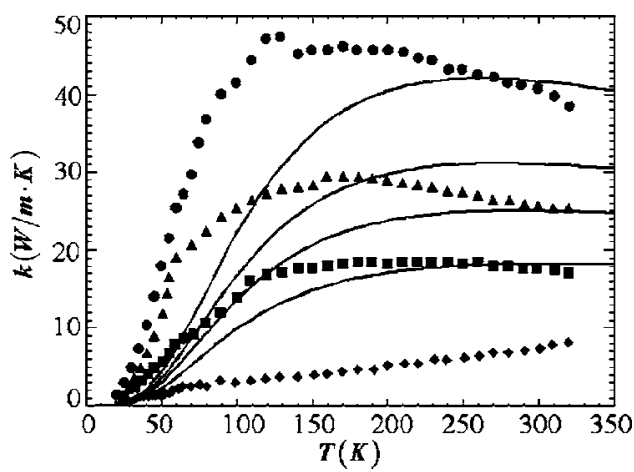

FIG. 10. A comparison between the experimental measurements and the theoretical predictions (B model with $p=0$ ) of the lattice thermal conductivity of a silicon nanowire. Four diameters are investigated: $22,37,56$, and $115 \mathrm{~nm}$.

The difference, however, is small. At last, we present a comparison of the theoretical predictions (B model) with the experimental measurements made by $\mathrm{Li}$ et al. ${ }^{21}$ in Fig. 10. The maximum diameter investigated is $115 \mathrm{~nm}$. It is found from Fig. 9 that the deduction of lattice thermal conductivity by the boundary scattering is more than $40 \%(p=0)$ for this diameter. The present models therefore should work fine herein. Although quantitative differences are observed, the predicted and measured lattice thermal conductivities are on the same order of magnitude.

\section{CONCLUSION}

In the present investigation, the lattice thermal conductivity models for semiconductor nanowires are developed. The size confinement effects on the phonon dispersion relations, the phonon distribution, and the Debye temperatures are all examined. Moreover, an analytical expression is derived for the boundary scattering rate and two methods are proposed to include it in the lattice thermal conductivity model correctly. The proposed theory herein is considered more consistent and more complete than other similar existing theories.

The computational results show that the lattice thermal conductivity of nanowires increases with an increasing diameter and an increasing specular fraction. It is only weakly dependent on the temperature. Most of all, as the diameter is small (say, $20 \mathrm{~nm}$ ), the predicted thermal conductivity is about one order of magnitude lower than that of the bulk value, agreeing well with the experimental observations. Besides the enhanced boundary scattering effect and the reduced phonon velocities, the lowered Debye temperatures are also responsible for the decrease of the lattice thermal conductivity of nanowires.

\section{ACKNOWLEDGMENTS}

The support of this work by the National Science Council, Taiwan, R.O.C. under Contract No. NSC 94-2212-E-002046 and that by ITRI under Contract No. 0000108475 are gratefully acknowledged.

${ }^{1}$ S. Wisniewski, B. Staniszewski, and R. Szymanik, Thermodynamics of Nonequilibrium Processes, (PWN-Polish Scientific, Boston, 1976).

${ }^{2}$ W. S. Capinski, H. J. Maris, T. Ruf, M. Cardona, K. Ploog, and D. S. Katzer, Phys. Rev. B 59, 8105 (1999).

${ }^{3}$ R. Venkatasubramanian, E. Silvola, T. Colpitts, and B. O'Quinn, Nature (London) 413, 597 (2001).

${ }^{4}$ M. V. Simkin and G. D. Mahan, Phys. Rev. Lett. 84, 927 (2000).

${ }^{5}$ X. Y. Yu, L. Zhang, and G. Chen, Rev. Sci. Instrum. 67, 2312 (1996).

${ }^{6}$ A. Balandin and K. L. Wang, Phys. Rev. B 58, 1544 (1998).

${ }^{7}$ D. G. Cahill, K. Goodson, and A. Majumdar, J. Heat Transfer 124, 223 (2002).

${ }^{8}$ G. Chen and A. Shakouri, J. Heat Transfer 124, 242 (2002).

${ }^{9}$ P. G. Klemens, in Solid State Physics, edited by F. Seitz and D. Turnbull (Academic, New York, 1958), Vol. 7, p. 1.

${ }^{10}$ A. Khitun, A. Balandin, and K. L. Wang, Superlattices Microstruct. 26, 181 (1999)

${ }^{11}$ N. Bannov, V. Aristov, and V. Mitin, Phys. Rev. B 51, 9930 (1995).

${ }^{12}$ J. M. Ziman, Electrons and Phonons (Oxford University Press, Clarendon, 2001).

${ }^{13}$ P. Hyldgaard and G. D. Mahan, Therm. Conduct. 23, 172 (1996).

${ }^{14}$ S. G. Walkauskas, D. A. Broido, K. Kempa, and T. L. Reinecke, J. Appl. Phys. 85, 2579 (1999).

${ }^{15}$ J. D. Chung, A. J. H. McGaughey, and M. Kaviany, J. Heat Transfer 126, 376 (2004)

${ }^{16}$ A. A. Kiselev, K. W. Kim, and M. A. Stroscio, Phys. Rev. B 62, 6896 (2000).

${ }^{17}$ J. Zou and A. Balandin, J. Appl. Phys. 89, 2932 (2001).

${ }^{18}$ A. Majumdar, J. Heat Transfer 115, 7 (1993).

${ }^{19}$ G. Chen, J. Heat Transfer 119, 220 (1997).

${ }^{20}$ G. Chen, Phys. Rev. B 57, 14958 (1998).

${ }^{21}$ D. Li, Y. Wu, P. Kim, L. Shi, P. Yang, and A. Majumdar, Appl. Phys. Lett. 83, 2934 (2003). 
Journal of Applied Physics is copyrighted by the American Institute of Physics (AIP). Redistribution of journal material is subject to the AIP online journal license and/or AIP copyright. For more information, see http://ojps.aip.org/japo/japcr/jsp 